\title{
Takayasu's Arteritis and Giant Cell (Temporal or Cranial) Arteritis
}

Key words: aortitis, aortitis syndrome, HLA antigen

Takayasu's arteritis (TA), of which the first case was described by Takayasu in 1905 (1), is a chronic inflammatory disease of unknown etiology that primarily involves the aorta, its major branches, and the pulmonary and coronary arteries. Synonyms such as aortic arch syndrome, pulseless disease and aortitis syndrome reflect this anatomic localization. TA appears to be most common in East Asia including Japan. According to a recent nationwide epidemiological survey, the total number of Japanese patients with TA in 1995 was estimated as 3,000 to 4,000 , showing a predominantly high female prevalence (male:female ratio 1:9.2) (2). TA is known for characteristically affecting a young female population, although the mean age of Japanese patients with TA has become older than previously described (2). In this issue of the Journal, a rare case of TA in a 69-year-old woman is reported (3).

See also p 69.

TA is also known for ethnic differences in the disease patterns and the associations of HLA antigens. For example, according to a new classification of the angiographic findings for TA (4), types I and II in Japanese patients are statistically significantly more common compared to those in Indian patients (5). In contrast, Indian patients have a rather high frequency of type IV which involves only the abdominal aorta and its branches. Concerning HLA antigens, although Japanese patients with TA are known to be associated with HLAB52 and B39 (6), it has been suggested that HLA-B39 might be associated with the involvement of the abdominal aorta in TA (7). The case of TA associated with HLA-B52 and its haplotype reported in this issue (3) is thought to represent type $\mathrm{V}$, which is characterized by the involvement of aortic arch, thoracic aorta, abdominal aorta and their branches, according to the above angiographic classification.

On the other hand, giant cell (temporal or cranial) arteritis (GCA), which was described by Hutchinson (8) in 1890 and by Horton et al (9) in 1932, has been recognized as a clinicopathological entity characterized as granulomatous temporal arteritis of the elderly. The incidence is likely high in Northern European countries, but it is low in Japan. A nationwide epidemiological survey by the collaborative study of the Research Committee on Intractable Vasculitis Syndrome and Epidemi- ology of Intractable Diseases sponsored by the Ministry of Health and Welfare of Japan was conducted for Japanese patients with GCA in 1998 (10). The total number of patients with GCA treated in 1997 was estimated to be 690 (range 400980), showing a weak predilection for women (male female ratio; $1: 1.8$ ) and a high incidence in elderly patients (mean age; 71.8 years old, range; $35-97$ years old). Recently, it was noted that GCA produced a distinct spectrum of clinical manifestations and often occured without involvement of the cranial arteries, indicating that a variant of GCA exists in which vasoocclusive disease of the large arteries of the upper extremities dominates the clinical presentation (11). The GCA variant with the involvement of the subclavian, axillary and brachial arteries could be distinguished from classic cranial arteritis and could occur in the absence of temporal arterial involvement, even though polymyalgia rheumatica occurred with a similar frequency in both groups of the patients with the GCA variant (28\%) and those with classic cranial arteritis (20\%). Furthermore, the GCA variant was more frequent in females, and affected slightly younger people. Both TA and GCA exhibit a strong preference for elastic arteries of larger caliber (12). TA often also involves the subclavian-axillary and proximal brachial arteries in a pattern indistinguishable from the GCA variant. These findings raise the question as to whether or not the GCA variant is different from TA. However, there are differences in the associations of HLA antigens between TA and GCA, apart from the differences in age at onset of both diseases and the lack of the involvement of temporal arteries in TA. It has been noted that patients with GCA are associated with HLA-DR4 $(13,14)$, although there is no data regarding the association of HLA in Japanese patients with GCA. Furthermore, it was reported that the patients with the GCA variant were associated with HLA-DRB $1 * 0404$, and this finding distinguished the above patients from those with classic cranial arteritis who were associated with HLA-DRB1*0401 (11). It is well known that there are differences in the frequencies of HLA-DRB 1 alleles among ethnic groups. Both HLADRB $1 * 0401$ and DRB $1 * 0404$ of which the alleles are components of the DR4 gene, are less frequent in the Japanese population, suggesting that this finding may contribute to the low prevalence of GCA in Japan.

Thus, while there is a possibility that some HLA alleles might be related to the disease patterns of TA and GCA, there might be differences in the associations between the disease patterns and HLA alleles according to race. Further studies on HLA alleles according to the disease patterns of TA and GCA in Japa- 
nese patients will be required to ascertain whether or not the GCA variant can be distinguished from TA with involvement of the aortic arch and its branches such as the subclavian, axillary, and brachial arteries, regardless of age at onset.

\section{Hiroshi Hashimoto, MD} Juntendo University, School of Medicine, Department of Internal Medicine and Rheumatology, 2-1-1, Hongo, Bunkyo-ku, Tokyo 113-8421

\section{References}

1) Takayasu M. A case with peculiar changes of the retinal central vessels. Acta Soc Ophthalmol Jpn 12: 554-555, 1908 (in Japanese).

2) Research Committee on Intractable vasculitis. Aortitis syndrome (Takayasu's arteritis). in: Guideline for Diagnosis and Treatment of Intractable Diseases (1). Research on Specific Diseases of the Ministry of Health and Welfare of Japan, Ed. Roppoushuppannsha, Tokyo, 1997: 134 140 (in Japanese).

3) Fukaya E, Miyata M, Takahashi A, et al. Takayasu's arteritis in a 69-yearold woman. Intern Med 39: 69-72, 2000.

4) Hata A, Noda M, Moriwaki R, Numano F. Angiographic findings of Takayasuarteritis; new classification. Int J Cardiol 54 Suppl: S155-S163, 1996.

5) Numano F. Differences in clinical presentation and outcome in different countries for Takayasu's arteritis. Curr Opin Rheumatol 9: 12-15, 1997.
6) Kimura A, Kitamura H, Date Y, Numano F. Comprehensive analysis of HLA genes in Takayasu arteritis in Japan. Int J Cardiol 54 Suppl: S61S69, 1996.

7) Numano F. HLA-B39 might play an important role in the pathogenesis of Takayasu arteritis. 1996 Annual Report of Research Committee on Intractable Vasculitis spon'sored by the Ministry of Health and Wefare of Japan (Chairman; Hashimoto H), 1997: 31-35 (in Japanese).

8) Hutchinson J. Diseases of the arteries. Arch Surg 1: 325-326, 1890.

9) Horton BT, Magath TB, Brown GE. An undescribed form of arteritis of the temporal vessels. Mayo Clin Proc 7: 700-701, 1932.

10) Matsumoto $Y$, Nakayama $T$, Ohno $Y$, et al. Nationwide epidemiological survey of refractory vasculitis (anti-neutrophil cytoplasmic antibody (ANCA) -associated vasculitis, anti-phospholipid syndrome and temporal arteritis) in Japan. 1998 Annual Report of Research Committee on Intractable Vasculitis sponsored by the Ministry of Health and Wefare of Japan (Chairman; Hashimoto H), 1999: 15-23 (in Japanese).

11) Brack A, Martinez-Taboada V, Stanson A, Goronzy JJ, Weyand CM. Disease pattern in cranial and large-vessel giant cell arteritis. Arthritis Rheum 42: 311-317, 1999.

12) Kerr GS, Hallahan CW, Giordano J, et al. Takayasu arteritis. Ann Intern Med 120: 919-929, 1994.

13) Barrier J, Bignon JD, Soulillou JP, Grolleau J. Increased prevalence of HLA-DR4 in giant-cell arteritis. N Engl J Med 305: 104-105, 1981 (letter).

14) Wernick R, Davey M, Bonafede P. Familial giant cell arteritis: report of an HLA-typed sibling pair and a review of the literature. Clin Exp Rheumatol 12: 63-66, 1994. 